\title{
The Detrimental Effect of Adversity on HIV Outcomes in the Era of Combined Anti-Retroviral Therapy*
}

\author{
Corrado Cancedda ${ }^{1,2,3+}$, Katherine S. Eddens ${ }^{4 \prime}$, Olumuyiwa Adebona ${ }^{2}$, Revathi Ravi ${ }^{5}$, \\ Enbal Shacham ${ }^{4}$, Edgar Turner Overton ${ }^{6}$
}

${ }^{1}$ Division of Global Health Equity, Brigham and Women's Hospital, Harvard Medical School, Boston, USA; ${ }^{2}$ Department of Global Health and Social Medicine, Harvard Medical School, Boston, USA; ${ }^{3}$ Partners In Health, Boston, USA; ${ }^{4}$ George Warren Brown School of Social Work, Washington University in St. Louis, St. Louis, USA; ${ }^{5}$ Texas Tech University Health Sciences Center, Lubbock, USA; ${ }^{6}$ Division of Infectious Diseases, University of Alabama at Birmingham, Birmingham, USA.

Email: ${ }^{\dagger}$ ccancedda@pih.org

Received October $2^{\text {nd }}, 2012$; revised October $28^{\text {th }}, 2012$; accepted November $14^{\text {th }}, 2012$

\begin{abstract}
Socially disadvantaged individuals with HIV experience unacceptably high rates of AIDS and death. The authors developed an adversity index to quantify the impact of demographic and psychosocial factors on HIV care. This retrospective cohort study included 431 participants with HIV on combined antiretroviral therapy between 2000 and 2005. Poor outcome was defined as progression to AIDS, death, virologic failure, or CD4 $<200$ cells $/ \mu 1$ at six months. The index utilized eight demographic and psychosocial variables independently associated with poor outcome. Exposure to higher numbers of variables in the adversity index significantly increased the rates of poor outcome from 14\% (exposure to no variables) to $100 \%$ (exposure to seven variables). The odds of poor outcome decreased but remained significant after adjusting for adherence-defining variables, indicating a possible mediating effect of poor adherence. Durably effective therapeutic strategies must include addressing adverse demographic and psychosocial factors affecting people living with HIV.
\end{abstract}

Keywords: HIV; Outcomes; Psychosocial; Adversity; Adherence

\section{Introduction}

The past two decades have witnessed dramatic changes in the epidemiology and disease progression associated with HIV infection. Once considered to be a death sentence, the development of active chemotherapy against the HIV virus has transformed its clinical course from that of a fatal illness to a chronic, manageable condition $[1,2]$. In the United States (US), morbidity and mortality from HIV dropped significantly towards the end of the twentieth century and have continued to drop in the first decade of the 21 st century. This trend has been associated with increased access to combined active antiretroviral therapy (cART) [3-9]. Unfortunately, the rate of the decline in HIV-related morbidity and mortality in the US

\footnotetext{
"Data pertaining to this manuscript was presented at: The International AIDS Conference in Mexico City from August $3^{\text {rd }}-8^{\text {th }}, 2008$; and the American Public Health Association's Committee on Affiliates midyear meeting in Washington DC from June $27-29^{\text {th }}, 2010$. Conflicts of Interests and Source of funding: The authors received funding from Bristol-Meyers Squibb Virology Fellows Research Training Program, which did not present any conflicts of interest.

"Both authors have contributed equally to the manuscript.

${ }^{\dagger}$ Corresponding author.
}

has not been uniform across all people living with HIV (PLWH) [7,9-11].

It has become increasingly clear that outcomes for PLWH are influenced by a host of other factors, separate from the timely initiation of cART [6,12-14]. In the US, women on cART are more likely to fail treatment compared to men on the same medications [7,13,15-18]. Survival and infection rates of PLWH also differ significantly among ethnicities in the US, with African Americans bearing the majority of deaths and new infections $[7,11,15,17]$. These differences seem to be related to adverse psychosocial factors more prevalent within specific demographic groups, compared to the general population $[11,16,17,19,20]$. Psychiatric disorders, particularly depression and substance abuse, are strongly associated with poorer quality of life and response to cART among PLWH [9,11,15,18,21-23]. Other psychosocial factors associated with adverse outcome of cART include low household income, unemployment, living in a single parent household, and homelessness [7,15,20,24,25]. Thus, while clinical stage at diagnosis and access to cART are essential determinants of outcome in PLWH, additional demographic (gender, ethnicity) and psychosocial factors 
to which PLWH are exposed in their daily lives play an equally important role in their response to cART $[12,18$, 24].

In recognition of the significant morbidity and mortality burden associated with HIV infection, the US Government in 2010 introduced the National HIV/AIDS Strategy which is aimed at: 1) reducing the number of people who become infected with HIV; 2) increasing access to care and optimizing outcomes for PLWH; and 3) reducing HIV-related health disparities [26]. The attainment of these objectives will require the early identification of vulnerable patients who are exposed to adverse psychosocial factors, are more likely to fail cART, and will benefit from a multidisciplinary patient-centered treatment plan [24,27-29]. Yet presently, there is a limited availability of screening tools to identify such patients at point of care and to monitor them while under treatment.

This study was therefore carried out to assess the relationship between different demographic and psychosocial factors and outcomes among a cohort of PLWH receiving care at an outpatient HIV clinic, and to develop an adversity index to identify and monitor patients at risk of poor response to cART.

\section{Methods}

\subsection{Sample}

A retrospective study was conducted among a cohort of PLWH who entered care at Washington University HIV Clinic between January 2000 and July 2005. The study population included all patients who were followed for at least six months, and who commenced cART during this time period. Women who initiated cART exclusively for prevention of mother to child transmission and discontinued their antiretroviral medications after delivery were excluded from the study. The data were abstracted from the medical and case management records of each individual. This study was approved by Washington University Human Research Protection Office.

\subsection{Outcomes of Interest}

This study was primarily interested in any poor outcome, defined by any one of the following after a minimum period of six months under care: 1) death; 2) development of a new AIDS-defining condition while under care; 3) $\mathrm{CD} 4<200$ cells $/ \mu 1$ at the time of the last recorded visit; or 4) viral load $>400$ copies/ml at the time of the last recorded visit. The secondary outcomes of interest were clinical failure and laboratory failure. Clinical failure was defined as having either outcome 1) or 2) while under care, regardless of CD4 count and viral load at the time of the last recorded visit. Laboratory failure was defined as having either outcome 3 ) or 4) in the absence of death or development of a new AIDS-defining condition while under care.

\subsection{Psychosocial Variables}

Twenty different psychosocial and demographic variables were selected as possible determinants of primary and secondary outcomes, based on a review of the literature. Related variables were grouped into five different domains, as shown in Table 1(a): 1) demographic factors; 2) economic hardship; 3) familial stress; 4) substance abuse; 5) other mental health problems. The authors finalized definitions for each variable prior to record review.

\subsection{Covariates}

Other variables potentially associated with demographic and psychosocial variables and with outcomes of interest were introduced into the analysis as covariates. These covariates were grouped into four domains, as shown in Table 1(b): 1) access to and utilization of care; 2) adherence to cART; 3 ) advanced HIV at baseline; and 4) other comorbidities. Access to and utilization of care was inferred by clinic attendance $(\geq 3$ visits per year versus $<3$ visits per year). Poor adherence was defined as having any of the following while under care: self-reported poor adherence or development of one or more new drugspecific genotype mutation despite being on cART. Advanced HIV at baseline was defined as having any one of the following at the time of the first recorded visit: CD4 count $<100$ cells $/ \mu$, having $\geq 1$ AIDS-defining conditions, having previously received $\geq 2$ cART regimens, or resistance to $\geq 2$ antiretroviral agents. The other comorbidities domain was defined as having one or more of the following: anemia (hemoglobin $<10 \mathrm{~g} / \mathrm{dl}$ ), chronic renal failure (creatinine clearance $<40 \mathrm{ml} / \mathrm{min}$ by Cockroft Gault equation), class III or IV heart failure, liver failure or decompensated cirrhosis (AST/ALT > $200 \mathrm{IU} / \mathrm{L}$, and/ or Alkaline Phosphatase $>400 \mathrm{IU} / \mathrm{L}$, and/or INR $>2+$ albumin $<2.5 \mathrm{~g} / \mathrm{dl}$, and/or documented cirrhosis on imaging studies), active HBV (reactive HBsAg), or chronic HCV infection (detectable plasma HCV RNA), or currently using tobacco.

\subsection{Statistical Analysis}

The $\chi^{2}$ statistic was used for categorical data analysis to compare individual adversity variables and outcomes of interest. A p-value of $<0.05$ was used to determine statistical significance. For highly correlated variables, the variable with the least amount of missing data was kept in the model, while the other variable was excluded from further analysis. In this model, only income and employment status displayed a high degree of co-linearity $(\mathrm{R}=0.685)$. The income variable encompassed a greater 
Table 1. (a) Characteristics of $431 \mathrm{HIV}+$ patients seen in an urban infectious disease clinic from 2000-2006; (b) Characteristics of $431 \mathrm{HIV}+$ patients seen in an urban infectious disease clinic from 2000-2006.

(a)

\begin{tabular}{c} 
Domains and variables \\
(defined as anytime during treatment, except where indicated) \\
\hline Demographics \\
Being a female \\
Being non-white \\
Age $<18$ years at time of diagnosis \\
Being a male having sex with males (MSM) \\
Economic hardship \\
Having an income $<\$ 10,000$ per year \\
Having a $<12$ years education level \\
Being unemployed \\
Being homeless or living in a shelter \\
Being incarcerated \\
Having an incarcerated partner \\
Familial stress \\
Being a single parent \\
Being diagnosed while pregnant \\
Having new children while under care \\
Active substance abuse \\
Abusing alcohol \\
Abusing cocaine \\
Abusing intravenous drugs \\
Abusing another substance \\
Maving another psychiatric disorder \\
Having depression money or drugs \\
\end{tabular}

proportion of the study participants $(\mathrm{N}=301)$ than employment status $(\mathrm{N}=286)$ and was incorporated into the model, while the unemployment variable was excluded from further analysis. Multivariate analyses were subsequently performed on all the psychosocial and demographic variables that were associated with the outcomes of interest during the $\chi^{2}$ analysis. Only variables that remained significantly associated with increased odds of primary and secondary outcomes were used in the adversity index. In order to maintain an adequate sample size, the education level was imputed when missing by running a regression of education on all psychosocial and demographic variables.

Ultimately, an adversity index with nine points (0 - 8) was created, with possession of any of the significant variables equivalent to one point. Points were summed up to determine the degree of adversity with higher total points indicating higher adversity. The authors then used (b)

Domains and covariates
(defined as anytime during treatment, except where indicated)
Access to care
Poor attendance ( $<3$ visits per year)
Adherence to cART
Self-reported poor cART adherence
Development of $\geq 1$ new mutations on HAART
Advanced disease at baseline
CD4 count $<100$ cells $/ \mu 1$
Viral load greater than 400 copies $/ \mathrm{ml}$
AIDS defining illness at baseline
$\geq 2$ cART regimens at baseline
Resistance to $\geq 2$ cART regimens
Comorbidities
Tepatitis C Virus (HCV) infection
Hepatitis B Virus (HBV) infection
Anemia (hemoglobin $<10 \mathrm{~g} / \mathrm{dl}$ )
Heart disease (class III-IV heart failure)
Renal insufficiency (creatinine clearance $<40 \mathrm{ml} / \mathrm{min}$ )

multivariate logistic regression to obtain estimates of the odds of any poor outcome, clinical failure, and laboratory failure for the adversity index alone, and to estimate the adjusted odds of these outcomes of interest when including the influence of covariates on the relationship between the adversity index and the outcomes of interest. Finally, the authors evaluated the relationship of cART adherence as a mediator between the adversity index and primary and secondary outcomes. All statistical analyses were performed using SPSS (version 16.0) statistical software [30].

\section{Results}

Between January 2000 and July 2005, 593 adult HIVinfected participants entered care at Washington University HIV Clinic. Eighty-nine participants (15\%) did not meet inclusion criteria and were excluded from the study. 
Participants in this group were more likely to be nonwhite, female, 18 - 24 years of age, have less than high school education, be diagnosed during pregnancy, and have a new child while under care. The majority of these patients were pregnant women initiating cART to prevent vertical transmission. Seventy-three participants [12\%] with incomplete records were also excluded from the study. For 153 participants, education data were missing and imputed by statistical analysis. Participants with missing education data differed from those with education data only by their increased likelihood of using tobacco.

A total of 431 participants $(73 \%)$ were included in the study. The majority of the cohort was male $(68 \% ; \mathrm{N}=$ $292)$, non-white $(62 \% ; \mathrm{N}=267)$, unemployed $(66 \% ; \mathrm{N}=$ $286)$, and reported annual incomes of $<\$ 10,000(70 \%$; $\mathrm{N}=301)$. Approximately $10 \%$ of the sample reported being homeless or living in a shelter. About one-fifth of the cohort was a single parent $(\mathrm{N}=92)$ and $9 \%(\mathrm{~N}=38)$ were diagnosed with HIV while pregnant. Rates of alco- hol use occurred among about $15 \%$ of the sample. High rates of depression were found among $41 \%(\mathrm{~N}=178)$ of the cohort.

Univariate and multivariate logistic regression were used to calculate the odds of any poor outcome, clinical failure, and laboratory failure for each of the demographic and psychosocial variables (Table 2). The variables that remained independently associated with in creased odds of primary or secondary outcomes included: 1) non-white ethnicity; 2) income $<\$ 10,000$ per year; 3) incarceration; 4) being a single parent; 5) having new children while under care; 6) alcohol abuse; 7) cocaine abuse; and h) having other psychiatric disorders. These variables were all included in the adversity index. The authors calculated the adversity index for each of the participants based on the number of demographic and psychosocial variables that were associated with each participant. The mean adversity point sum was $2.15(\mathrm{SD}=$ 1.40), with a range from 0 - 7 (Figure 1).

A one-point increase in adversity score was associated

Table 2. Estimated odds ratios (OR) and 95\% confidence intervals (CI) resulting from multiple logistic regression analysis of any poor outcome, clinical failure and laboratory failure.

\begin{tabular}{|c|c|c|c|}
\hline \multirow{2}{*}{ Variables } & Any poor outcome & Clinical failure & Laboratory failure \\
\hline & OR $(95 \% \mathrm{CI})$ & OR $(95 \% \mathrm{CI})$ & OR $(95 \% \mathrm{CI})$ \\
\hline \multicolumn{4}{|l|}{ Demographics } \\
\hline Being a female & $0.55(0.30,1.01)$ & $0.73(0.32,1.67)$ & $0.70(0.36,1.35)$ \\
\hline Being non-white & $2.25(1.34,3.77)^{*}$ & $1.18(0.58,2.40)$ & $2.19(1.23,3.88)^{*}$ \\
\hline Age $<18$ at time of diagnosis & $2.92(0.89,9.55)$ & $2.33(0.62 .8 .77)$ & $1.75(0.59,5.16)$ \\
\hline \multicolumn{4}{|l|}{ Economic hardship } \\
\hline Annual income $<\$ 10,000$ & $3.33(1.93,5.75)^{*}$ & $8.58(2.55,28.86)^{*}$ & $1.64(0.91,2.97)$ \\
\hline Having $<12$ years education & $1.04(0.54,1.97)$ & $0.81(0.36,1.82)$ & $1.22(0.64,2.32)$ \\
\hline Homeless/living in a shelter & $1.53(0.70,3.37)$ & $1.51(0.63,3.66)$ & $1.21(0.56,2.64)$ \\
\hline Being incarcerated & $1.02(0.43,2.40)$ & $0.16(0.03,0.74)$ & $2.52(1.09,5.82)^{*}$ \\
\hline \multicolumn{4}{|l|}{ Familial stress } \\
\hline Being a single parent & $2.67(1.36,5.22)^{*}$ & $2.96(1.20,7.30)^{*}$ & $1.51(0.75,3.05)$ \\
\hline Having new children during care & $1.31(0.65,2.67)$ & $0.40(0.14,1.14)$ & $2.19(1.09,4.41)^{*}$ \\
\hline \multicolumn{4}{|l|}{ Active substance abuse } \\
\hline Abusing alcohol & $0.67(0.34,1.30)$ & $2.42(1.13,5.19)^{*}$ & $0.31(0.14,0.70)^{*}$ \\
\hline Abusing cocaine & $2.72(1.29,5.77)^{*}$ & $1.21(0.50,2.92)$ & $2.58(1.22,5.48)^{*}$ \\
\hline Abusing another substance & $1.34(0.70,2.55)$ & $0.98(0.44,2.19)$ & $1.30(0.66,2.56)$ \\
\hline Trading sex for money or drugs & $0.17(0.01,2.19)$ & 0 & $0.27(0.02,4.49)$ \\
\hline \multicolumn{4}{|l|}{ Mental health issues } \\
\hline Having depression & $1.48(0.91,2.40)$ & $1.12(0.59,2.14)$ & $1.24(0.74,2.10)$ \\
\hline Having another psychiatric disorder & $3.41(1.65,7.06)^{*}$ & $1.73(0.74,4.03)$ & $2.61(1.30,5.27)^{*}$ \\
\hline
\end{tabular}


with increased odds of both primary (cOR 1.90, 95\% CI $1.61-2.25$ ) and secondary outcomes (clinical failurecOR 1.47, 95\% CI 1.21 - 1.78; laboratory failure-cOR 1.64, 95\% CI 1.40 - 1.93), shown in Table 3. Even after adjusting for the covariates, the odds of any poor outcome (aOR 1.68, 95\% CI $1.34-2.09$ ) and laboratory failure (aOR 1.60, 95\% CI 1.28 - 1.98) with higher total points remained significant. Figure 2 shows the distribution of primary and secondary outcomes across levels of the adversity index, indicating that the proportion of patients responding poorly to cART grows with each increase in adversity index. One hundred percent of those who had the highest total points on the adversity index (7) experienced any poor outcome. Higher scores were also associated with higher odds of self-reported poor adherence (cOR 1.91, 95\% CI $1.61-2.28$ ) as well as the development of new mutations while under care (cOR 1.38,
95\% CI 1.17 - 1.63) (Table 4). These covariates were also strongly correlated with the primary and secondary outcomes, indicating a possible mediation of the relationship between adversity index and outcomes of interest by cART adherence. Covariates indicating the presence of other comorbidities were correlated with clinical failure, and after adjusting for these covariates the adversity index was not significantly associated with clinical failure.

\section{Discussion}

This data confirms that demographic and psychosocial factors have a profound influence on outcomes among PLWH $[13-14,20]$. Not only did the authors find a strong independent association between individual variables and any poor outcome, but the accumulation of multiple ad-

Table 3. Estimated odds ratios (OR) and 95\% confidence intervals (CI) resulting from multivariate logistic regression analysis of increasing adversity score and covariates on any poor outcome, clinical failure and laboratory failure.

\begin{tabular}{|c|c|c|c|}
\hline \multirow{2}{*}{ Variables } & Any poor outcome & Clinical failure & Laboratory failure \\
\hline & OR $(95 \% \mathrm{CI})$ & OR $(95 \% \mathrm{CI})$ & OR $(95 \% \mathrm{CI})$ \\
\hline \multicolumn{4}{|l|}{ Covariates } \\
\hline \multicolumn{4}{|l|}{ Access to care } \\
\hline Poor attendance & $1.13(0.53,2.42)$ & $0.87(0.33,2.32)$ & $1.10(0.53,2.27)$ \\
\hline \multicolumn{4}{|l|}{ Adherence to cART } \\
\hline Self-reported poor cART adherence & $6.19(3.32,11.54)^{*}$ & $6.37(2.71,14.98)^{*}$ & $2.57(1.40,4.69)^{*}$ \\
\hline New mutations & $2.67(1.41,5.08)^{*}$ & $0.93(0.41,2.09)$ & $2.23(1.19,4.15)^{*}$ \\
\hline CD4 count less than 100 & $2.12(1.08,4.15)^{*}$ & $0.75(0.30,1.88)$ & $1.46(0.74,2.86)$ \\
\hline Viral load greater than 4 & $2.29(1.25,4.19)^{*}$ & $3.10(1.24,7.75)^{*}$ & $1.31(0.72,2.36)$ \\
\hline AIDS at baseline & $0.69(0.36,1.32)$ & $1.92(0.80,4.61)$ & $0.65(0.33,1.27)$ \\
\hline Baseline mutations & $2.21(0.96,5.10)$ & $2.48(0.89,6.93)$ & $1.37(0.60,3.14)$ \\
\hline$>1$ cART regimen at baseline & $1.25(0.59,2.64)$ & $0.73(0.24,2.24)$ & $1.47(0.69,3.12)$ \\
\hline \multicolumn{4}{|l|}{ COMORBIDITIES } \\
\hline Anemia & $1.35(0.51,3.57)$ & $6.19(2.46,15.55)^{*}$ & $0.19(0.06,0.54)$ \\
\hline Renal insufficiency & $0.62(0.18,2.17)$ & $3.34(1.02,10.95)^{*}$ & $0.64(0.15,2.70)$ \\
\hline Heart disease & $0.89(0.29,2.73)$ & $4.35(1.30,14.51)^{*}$ & $0.11(0.01,0.89)$ \\
\hline $\mathrm{HCV}$ & $1.49(0.63,3.52)$ & $1.81(0.67,4.89)$ & $0.61(0.25,1.52)$ \\
\hline HBV & $2.93(1.07,8.00)^{*}$ & $2.30(0.78,6.84)$ & $1.80(0.69,4.69)$ \\
\hline Tobacco & $0.73(0.42,1.26)$ & $0.99(0.48,2.08)$ & $0.82(0.48,1.42)$ \\
\hline Model $\mathrm{R}^{2}$ & 0.47 & 0.43 & 0.30 \\
\hline
\end{tabular}


Table 4. Odds of poor adherence cART by adversity index.

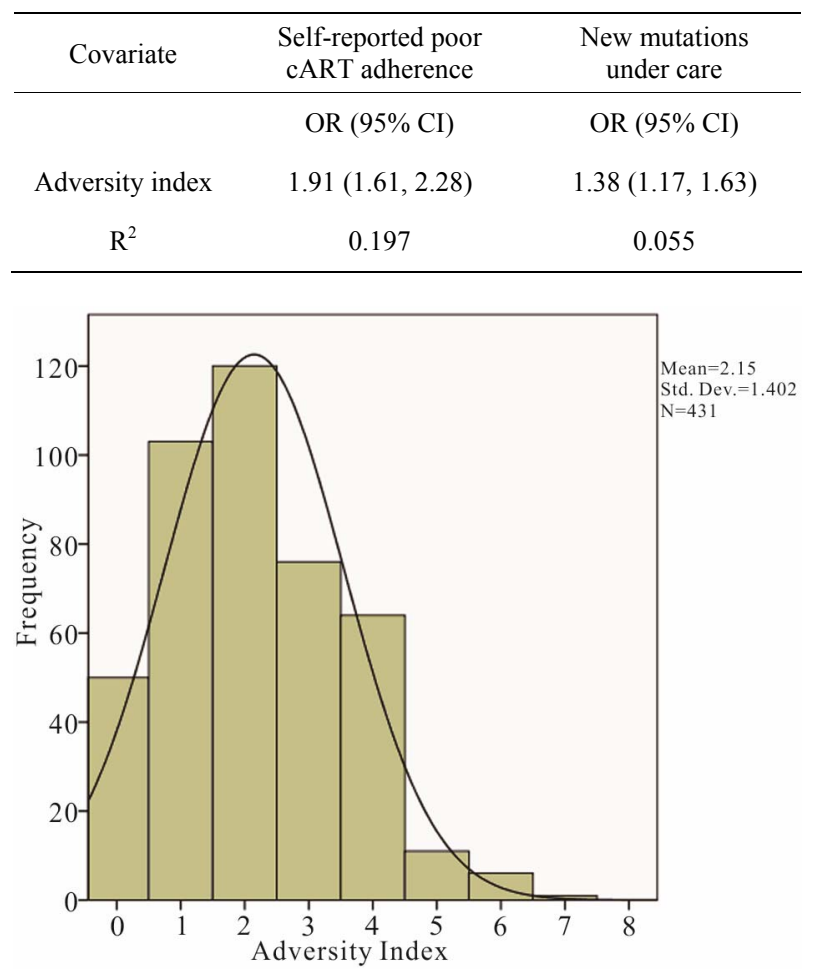

Figure 1. Distribution of adversity index in $431 \mathrm{HIV}+$ patients seen in an urban infectious disease clinic from 2000 2006.

verse demographic and psychosocial variables greatly reduced the odds of cART success. In fact, the addition of a single variable that shifted a person to a higher point sum on the adversity index increased the odds of any poor outcome by $68 \%$. While this study failed to find independent associations with other variables that have been previously linked to poor outcomes such as low education and homelessness [20,24,31-32], these variables were more prevalent in participants with higher total points in the adversity index. This finding may have relevance in clinical practice since such variables may serve as proxies for underlying measures of social disadvantage associated with poor outcome and may point caregivers towards their pursuit.

These findings demonstrate that the majority of participants were exposed to a significant number of adverse demographic and psychosocial variables, with 35\% of participants being exposed to three or more simultaneously. The progression of the adversity index was determined by the addition of just one variable, yet the associated increase in the odds of poor outcome was substantial. This finding suggests that in the case of psychosocial variables, a single discreet life event (like becoming a single parent) can easily alter the balance of care and that specific interventions addressing the event may have a major impact in restoring or improving such balance.

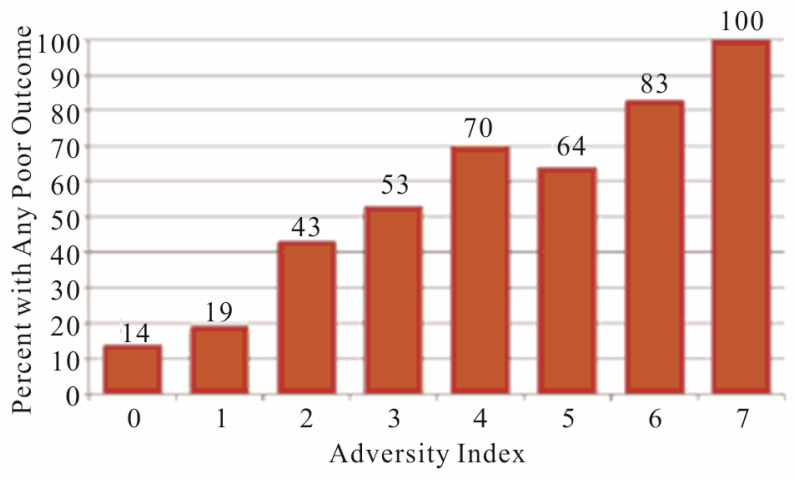

(a)

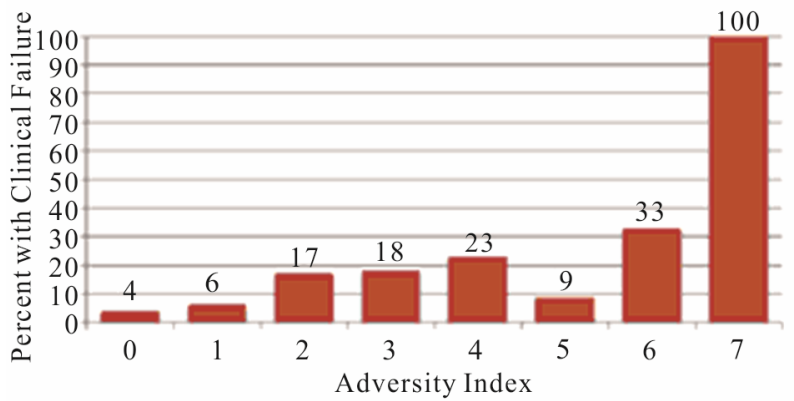

(b)

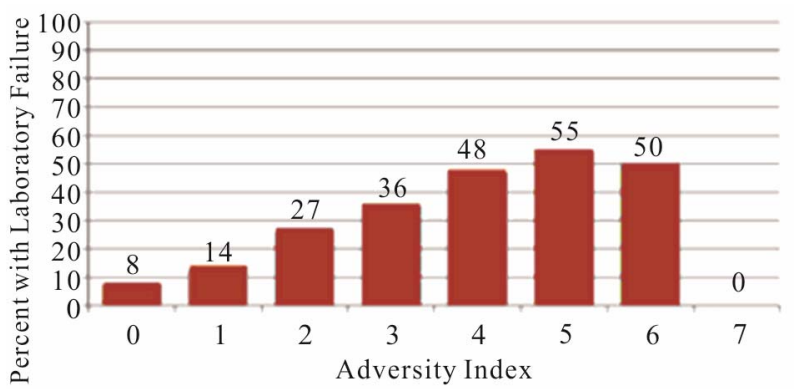

(c)

Figure 2. Distribution of primary (a) and secondary (b), (c) outcomes of interest across the adversity index.

In this cohort, limited access to care, having advanced $\mathrm{HIV}$ at the time of CART initiation, and poor adherence to cART all contributed to primary and secondary outcome. Participants with higher total points in the adversity index were more likely to miss follow up appointments with their care-givers, initiate cART at a more advanced HIV stage, and to miss cART doses while on treatment. However, even after adjusting for covariates within these domains, the odds of poor outcome remained significantly higher for patients with higher total points in the adversity index indicating that the accumulation of adverse demographic and psychosocial variables has an additional detrimental effect on HIV care. The definition of poor adherence in this study (which relied heavily on self-reporting by participants) may have led to an under-estimation of its prevalence within the 
cohort, but it is also possible that other covariates yet to be identified contributed to cART failure among participants with higher total points in the adversity index.

A particularly interesting finding in this study was the significant association between higher total points in the adversity index and increased odds of poor adherence to cART, despite the fact that few participants had a score greater than six, and none had a score of eight.

Among PLWH, adherence is a strong predictor of response to cART [32-37]. Failure of first-line cART leads to the initiation of second- and third-line regimens, which are often more expensive [38,39]. The adversity index developed in this study could help identify patients more likely to miss cART doses as a result of exposure to adverse psychosocial factors and be an important adjunct to the management of PLWH, both prior to the initiation of cART and during treatment. Patient-centered care models for PLWH ensure early initiation of and adherence to cART by addressing adverse psychosocial factors and as a result reduce healthcare associated costs by limiting hospitalizations and the initiation of alternative cART regimens [40-45]. Within such a framework, an adversity index such as the one developed for this study will help caregivers identify high-risk patients and establish "wraparound" services addressing the psychosocial factors most likely to negatively impact adherence and the odds of a successful response to cART.

There are limitations to these findings. This study was conducted within a single urban clinic cohort within a Mid-Western city, which reduces the extent to which the results presented above can be extrapolated to the other HIV-infected populations. The retrospective nature of the study limited the types of data that the authors were able to collect as well as the options for the study design; yet it did provide an opportunity to collate medical and case manager records to create a more comprehensive impression of each patient. For 153 patients, education data was unavailable and had to be imputed by statistical analysis. One cannot therefore entirely rule out the importance of the education level variable in the adversity index, particularly since previous studies have found associations between lower education level and cART failure $[15,20$, 46]. The authors considered the development of a multiitem additive scale as an alternative approach to quantify the impact of demographic and psychosocial variables within different domains 1) demographic factors 2), economic hardship, 3) familial stress, 4) substance abuse, 5) other mental health problems on HIV care. However, the development of such a scale reduced the strength of the association between the domains and poor outcome, probably because within each domain were also included variables that were not independently associated with the outcomes of interest.

While AIDS Drug Assistance Programs have until re- cently broadened the ability for PLWH to receive therapy regardless of health insurance status, the widening disparity in outcomes of interest observed among PLWH has demonstrated that this is not enough $[12,13]$. Helping patients overcome barriers to care by providing comprehensive "wrap-around" services is a well-established practice in developing countries and has been implemented in the US as well with remarkable successes [42, $47,48]$. Some of these services that have been enacted to overcome barriers to access and adherence include coverage of transportation fees, provision of day-care options for single mothers, close psychiatric follow up for patients needing mental health care, substance abuse treatment, and regular home visits by community health workers.

HIV/AIDS is now a disease driven by social inequalities and comprehensive interventions that demonstrate how the adverse demographic and psychosocial factors affect PLWH are a fundamental part of a truly effective therapeutic strategy. The development of a tool that allows early identification and close monitoring of patients at risk for poor adherence may positively impact their long-term response to cART. The authors propose that the adversity index presented in this study may play such a role in the care of PLWH.

\section{REFERENCES}

[1] C. Liu, L. Johnson, D. Ostrow, et al., "Predictors for Lower Quality of Life in the HAART Era among HIV-Infected Men," Journal of Acquired Immune Deficiency Syndromes, Vol. 42, No. 4, 2006, pp. 470-477. doi:10.1097/01.qai.0000225730.79610.61

[2] C. J. Colvin, "HIV/AIDS Chronic Diseases and Globalization," Globalization and Health, Vol. 7, No. 31, 2011. http://www.globalizationandhealth.com/content/7/1/31

[3] CDC, "Epidemiology of HIV/AIDS - United States, 19812005," Morbidity and Mortality Weekly Report, Vol. 55, No. 21, 2006, pp. 589-592.

[4] M. Thompson, J. Aberg, P. Cahn, et al., "Antiretroviral Treatment of Adult HIV Infection: 2010 Recommendations of the International AIDS Society-USA Panel," Journal of the American Medical Association, Vol. 304, No. 3, 2010, pp. 321-333. doi:10.1001/jama.2010.1004

[5] D. Lai and R. J. Hardy, "An Update on the Impact of HIV/AIDS on Life Expectancy in the United States," AIDS, Vol. 18, No. 12, 2004, pp. 1727-1740. doi:10.1097/01.aids.0000131383.15232.95

[6] F. J. Palella, K. M. Delaney, A. C. Moorman, et al., "Declining Morbidity and Mortality among Patients with Advanced Human Immunodeficiency Virus Infection," The New England Journal of Medicine, Vol. 339, No. 6, 1998, pp. 405-406. doi:10.1056/NEJM199808063390612

[7] J. M. Karon, P. L. Fleming, R. W. Steketee, et al., "HIV in the United States at the Turn of the Century: An Epidemic in Transition," American Journal of Public Health, 
Vol. 91, No. 7, 2001, pp. 1060-1068. doi:10.2105/AJPH.91.7.1060

[8] E. L. Murphy, A. C. Collier, L. A. Kalish, et al., "Highly Active Antiretroviral Therapy Decreases Mortality and Morbidity in Patients with Advanced HIV Disease," Annals of Internal Medicine, Vol. 135, No. 1, 2001, pp. 1726.

[9] G. Chander, S. Himelhoch and R. D. Moore, "Substance Abuse and Psychiatric Disorders in HIV-Positive Patients Epidemiology and Impact on Antiretroviral Therapy," Drugs, Vol. 66, No. 6, 2006, pp. 769-789. doi:10.2165/00003495-200666060-00004

[10] CDC, "HIV Surveillance Report, 2009," Vol. 21, 2011. http://www.cdc.gov/hiv/surveillance/resources/reports/20 09report/

[11] K. Anastos, M. F. Schneider, S. J. Gange, et al., "The Association of Race, Sociodemographic, and Behavioral Characteristics with Response to Highly Active Antiretroviral Therapy in Women," Journal Acquired Immune Deficiency Syndromes, Vol. 39, No. 5, 2005, pp. 537-544.

[12] CDC, "Establishing a Holistic Framework to Reduce Iniquities in HIV, Viral Hepatitis, STDs and Tuberculosis in the United States," Department of Health and Human Services, Atlanta, 2010.

[13] E. Losina, B. Schackman, S. Sadownik, et al., "Racial and Sex Disparities in Life Expectancy Losses among HIV-Infected Persons in the United States: Impact of Risk Behavior, Late Initiation, and Early Discontinuation of Antiretroviral Therapy," Clinical Infectious Diseases, Vol. 49, No. 10, 2009, pp. 1570-1578. doi: $10.1086 / 644772$

[14] W. Pence, J. Ostermann, V. Kumar, et al., "The Influence of Psychosocial Characteristics and Race/Ethnicity on the Use, Duration, and Success of Antiretroviral Therapy," Journal Acquired Immune Deficiency Syndrome, Vol. 47, No. 2, 2008, pp. 194-201. doi:10.1097/QAI.0b013e31815ace7e

[15] E. Shacham, D. Nurutdinova, N. Onen, et al., "The Interplay of Sociodemographic Factors on Virologic Suppression among a US Outpatient HIV Clinic Population," AIDS Patient Care STDS, Vol. 24, No. 4, 2010, pp. 229235. doi:10.1089/apc.2009.0275

[16] A. L. Meditz, S. MaWhinney, A. Allshouse, et al., "Sex, Race and Geographic Region Influence Clinical Outcomes of Interest Following Primary HIV-1 Infection," Journal of Infectious Diseases, Vol. 203, No. 4, 2011, pp. 442-451. doi:10.1093/infdis/jiq085

[17] D. C. Lemly, B. E. Shepherd, T. Hulgan, et al., "Race and Sex Differences in Antiretroviral Therapy Use and Mortality among HIV-Infected Persons in Care," Journal of Infectious Diseases, Vol. 199, No. 7, 2009, pp. 991-998. doi:10.1086/597124

[18] D. A. Rastegar, M. I. Fingerhood and D. R. Jasinski, "Highly Active Antiretroviral Therapy Outcomes of Interest in a Primary Care Clinic," AIDS Care: Psychological and Socio-Medical Aspects of AIDS/HIV, Vol. 15, No. 2, 2003, pp. 231-237.

[19] H. D. Dean, C. B. Steele, A. J. Satcher, et al., "HIV/AIDS among Minority Races and Ethnicities in the United
States, 1999-2003," Journal of the National Medical Association, Vol. 97, No. 7S, 2005, pp. S5-S12.

[20] W. E. Cunningham, R. D. Hays, N. Duan, et al., "The Effect of Socioeconomic Status on the Survival of People Receiving Care for HIV Infection in the United States," Journal of Health Care for the Poor and Underserved, Vol. 15, No. 4, 2005, pp. 655-676. doi:10.1353/hpu.2005.0093

[21] C. Liu, K. Weber, E. Robison, et al., "Assessing the Effect of HAART on Change in Quality of Life among HIV-Infected Women," AIDS Research and Therapy, Vol. 3, No. 6, 2006. http://www.aidsrestherapy.com/content/3/1/6

[22] B. W. Pence, W. C. Miller, B. N. Gaynes, et al., "Psychiatric Illness and Virologic Response in Patients Initiating Highly Active Antiretroviral Therapy," Journal of Acquired Immune Deficiency Syndromes, Vol. 44, No. 2, 2007, pp. 159-166. doi:10.1097/QAI.0b013e31802c2f51

[23] G. Chander, B. Lau and R. D. Moore, "Hazardous Alcohol Use: A Risk Factor for Non-Adherence and Lack of Suppression in HIV Infection," Journal of Acquired Immune Deficiency Syndromes, Vol. 43, No. 4, 2006, pp. 411-417. doi:10.1097/01.qai.0000243121.44659.a4

[24] S. Lieb, R. G. Brooks, R. S. Hopkins, et al., "Predicting Death from HIV/AIDS: A Case-Control Study from Florida Public HIV/AIDS Clinics," Journal Acquired Immune Deficiency Syndromes, Vol. 30, No. 3, 2002, pp. 351-358. doi:10.1097/00042560-200207010-00012

[25] R. Goldstein, M. Johnson, M. Rotheram-Borus, et al., "Psychological Distress, Substance Use, and Adjustment among Parents Living with HIV," Journal of American Board of Family Practice, Vol. 18, No. 5, 2005, pp. 362373. doi: $10.3122 / \mathrm{jabfm} .18 .5 .362$

[26] The White House, "National HIV/AIDS Strategy of the United States," Washington DC, 2010.

[27] M. Mitka, "Patient-Centered Medical Homes Offer a Model for Better, Cheaper Health Care," Journal of the American Medical Association, Vol. 307, No. 8, 2012, pp. 770-771. doi:10.1001/jama.2012.180

[28] M. Davis-Michaud, R. Yurk, D. Lansky, et al., "Quality of Care for People with HIV/AIDS: Patients' Perspectives," HIV Clinical Trials, Vol. 5, No. 6, 2004, pp. 406415. doi:10.1310/GQ98-PGK7-0T97-47XH

[29] K. Stange, P. Nutting, W. Miller, et al., "Defining and Measuring the Patient-Centered Medical Home," Journal of General Internal Medicine, Vol. 25, No. 6, 2010, pp. 601-612. doi:10.1007/s11606-010-1291-3

[30] SPSS 16.0 Graduate Student Version [Computer Program], Chicago, 2007.

[31] A. Harzke, M. Ross and D. Scott, "Predictors of PostRelease Primary Care Utilization among HIV-Positive Prison Inmates: A Pilot Study," AIDS Care: Psychological and Socio-Medical Aspects of AIDS/HIV, Vol. 18, No. 4, 2006, pp. 290-301.

[32] J. Kowlaski, S. Gange, M. Schneider, et al., "Relationship of Injection Drug Use, Antiretroviral Therapy Resistance, and Genetic Diversity in the HIV-1 Pol Gene," Journal of Acquired Immune Deficiency Syndromes, Vol. 50, No. 4, 
2009, pp. 381-389. doi:10.1097/QAI.0b013e318198a619

[33] G. DeLorenze, C. Weisner, A. Tsai, et al., "Excessive Mortality among HIV-Infected Patients Diagnosed with Substance Use Dependence of Abuse Receiving Care in a Fully Integrated Medical Care Program," Alcoholism Clinical and Experimental Research, Vol. 35, No. 2, 2011, pp. 203-210. doi:10.1111/j.1530-0277.2010.01335.x

[34] S. Mannheimer, G. Friedland, C. Child, et al., "The Consistency of Adherence to Antiretroviral Therapy Predicts Biological Outcomes of Interest for Human Immunodeficiency Virus-Infected Persons in Clinical Trials," Clinical Infectious Diseases, Vol. 34, No. 8, 2002, pp. 1115-1121. doi:10.1086/339074

[35] B. Turner, "Adherence to Antiretroviral Therapy by Human Immunodeficiency Virus-Infected Patients," Journal of Infectious Diseases, Vol. 185, No. S2, 2002, pp. S143S151. doi:10.1086/340197

[36] F. Maggiolo, M. Airoldi, D. Hendrik, et al., "Effect of Adherence to HAART on Virologic Outcome and on the Selection of Resistance-Conferring Mutations in NNRTIor PI-Treated Patients," HIV Clinical Trials, Vol. 8, No. 5, 2007, pp. 282-292. doi:10.1310/hct0805-282

[37] M. Chesney, M. Morin and L. Sherr, "Adherence to HIV Combination Therapy," Social Science and Medicine, Vol. 50, No. 11, 2000, pp. 1599-1605. doi:10.1016/S0277-9536(99)00468-2

[38] B. Schackman, K. Gebo, R. Walensky, et al., "The Lifetime Cost of Current Human Immunodeficiency Virus Care in the United States," Medical Care, Vol. 44, No. 11, 2006, pp. 990-997. doi:10.1097/01.mlr.0000228021.89490.2a

[39] P. Volberding and S. Deeks, "Antiretroviral Therapy and Management of HIV Infection," The Lancet, Vol. 376, No. 9734, 2010, pp. 49-62. doi:10.1016/S0140-6736(10)60676-9

[40] T. Hoang, M. Goetz, E. Yano, et al., "The Impact of Integrated HIV Care on Patient Health Outcomes of Interest," Medical Care, Vol. 47, No. 5, 2009, pp. 560-567. doi:10.1097/MLR.0b013e31819432a0
[41] A. Gawande, "The Hot Spotters: Can We Lower Medical Costs by Giving the Neediest Patients Better Care?" The New Yorker, January 2011, pp. 40-51.

[42] H. Behforouz, P. Farmer and J. Mukherjee, "From Directly Observed Therapy to Accompagnateurs: Enhancing AIDS Treatment Outcomes of Interest in Haiti and in Boston," Clinical Infectious Diseases, Vol. 38, No. S5, 2004, pp. 429S-436S. doi:10.1086/421408

[43] M. Mitka, "Patient-Centered Medical Homes Offer a Model for Better, Cheaper Care," Journal of the American Medical Association. Vol. 307, No. 8, 2012, pp. 770771. doi:10.1001/jama.2012.180

[44] R. McLellan, B. Sherman, R. Loeppke, et al., "Optimizing Healthcare Delivery by Integrating Workplaces, Homes and Communities: How Occupational and Environmental Medicine can Serve as a Vital Connecting Link between Accountable Care Organizations and the PatientCentered Medical Home," Journal of Occupational and Environmental Medicine, Vol. 54, No. 4, 2012, pp. 504 512. doi:10.1097/JOM.0b013e31824fe0aa

[45] E. Fisher and S. Shortell, "Accountable Care Organizations: Accountable for What, to Whom and How," Journal of the American Medical Association, Vol. 304, No. 15, 2010, pp. 1175-1176. doi:10.1001/jama.2010.1513

[46] L. Marc, M. Testa, A. Walker, et al., "Educational Attainment and Response to HAART during Initial Therapy for HIV-1 Infection," Journal of Psychosomatic Research, Vol. 63, No. 2, 2007, pp. 207-216. doi:10.1016/j.jpsychores.2007.04.009

[47] J. Kim, P. Farmer, "AIDS in 2006-Moving toward One World, One Hope?" New England Journal of Medicine, Vol. 355, No. 7, 2006, pp. 645-647. doi:10.1056/NEJMp068166

[48] J. Mukherjee, L. Ivers, F. Leandre, et al., "Antiretroviral Therapy in Resource-Poor Settings. Decreasing Barriers to Access and Promoting Adherence," Journal Acquired Immune Deficiency Syndrome, Vol. 355, No. 7, 2006, pp. 123S-126S. doi:10.1097/01.qai.0000248348.25630.74 